Supplement of Earth Syst. Dynam. Discuss., 6, 1129-1162, 2015

http://www.earth-syst-dynam-discuss.net/6/1129/2015/

doi:10.5194/esdd-6-1129-2015-supplement

(C) Author(s) 2015. CC Attribution 3.0 License.

(c) (1)

$\begin{array}{r}\text { Earth System } \\ \text { Dynamics } \\ \hline \text { Discussions }\end{array}$

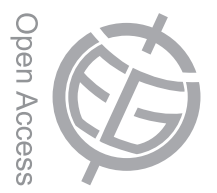

Supplement of

\title{
Potential impact of climate and socioeconomic changes on future agricultural land use in West Africa
}

\section{K. F. Ahmed et al.}

Correspondence to: K. F. Ahmed (kfa09002@engr.uconn.edu)

The copyright of individual parts of the supplement might differ from the CC-BY 3.0 licence. 
Table S1: Present-day (SPAM 2005) and the LandPro-projected future (mid-21 ${ }^{\text {st }}$ century) average crop area coverage in the West African countries.

\begin{tabular}{|l|c|c|c|}
\hline \multirow{2}{*}{ Country } & Present-day coverage & \multicolumn{2}{|c|}{ Future coverage (\%) } \\
\cline { 3 - 4 } & $\mathbf{( \% )}$ & MIROC-driven climate & CESM-driven climate \\
\hline Benin & 19.17 & 56.51 & 60.08 \\
\hline Burkina Faso & 20.38 & 42.96 & 37.05 \\
\hline Gambia & 31.34 & 76.31 & 70.57 \\
\hline Ghana & 19.12 & 41.35 & 52.03 \\
\hline Guinea & 6.83 & 40.27 & 42.92 \\
\hline Guinea-Bissau & 9.42 & 38.45 & 41.25 \\
\hline Ivory Coast & 13.27 & 27.65 & 37.62 \\
\hline Mali & 4.97 & 12 & 10.88 \\
\hline Niger & 13.07 & 17.79 & 17.63 \\
\hline Nigeria & 39.44 & 84.46 & 80.87 \\
\hline Senegal & 14.58 & 45.78 & 42.28 \\
\hline Sierra Leone & 8.7 & 35.96 & 39.35 \\
\hline Togo & 31.62 & 51.53 & 60.85 \\
\hline
\end{tabular}


Table S2: Future average crop area coverage in the West African countries under the MIROCdriven climate as projected by the LandPro algorithm following three different orders of yield values in selecting the cropping area to optimize agricultural land use. Initial scenario (best case in land use optimization): descending order of yield; alternative scenario 01 (worst case): ascending order; alternative scenario 02 (intermediate case): random order.

\begin{tabular}{|l|c|c|c|}
\hline \multirow{2}{*}{ Country } & \multicolumn{3}{|c|}{ Future coverage (\%) } \\
\cline { 2 - 4 } & Best case & Worst case & Intermediate case \\
\hline Benin & 56.51 & 74.97 & 57.22 \\
\hline Burkina Faso & 42.96 & 57.68 & 52.38 \\
\hline Gambia & 76.31 & 91.45 & 84.27 \\
\hline Ghana & 41.35 & 56.75 & 45.92 \\
\hline Guinea & 40.27 & 70.42 & 47.27 \\
\hline Guinea-Bissau & 38.45 & 80.02 & 39.41 \\
\hline Ivory Coast & 27.65 & 50.08 & 29.22 \\
\hline Mali & 12.00 & 22.02 & 16.36 \\
\hline Niger & 17.79 & 17.81 & 17.82 \\
\hline Nigeria & 84.46 & 90.36 & 89.18 \\
\hline Senegal & 45.78 & 81.3 & 67.93 \\
\hline Sierra Leone & 35.96 & 71.79 & 39.03 \\
\hline Togo & 51.53 & 73.16 & 60.14 \\
\hline
\end{tabular}


Table S3: Future average crop area coverage in the West African countries under the MIROCdriven climate as projected by the LandPro algorithm following four different rankings of crops prioritized by the farmers to optimize agricultural land use. Rank 1: descending order of country-level crop deficit; rank 2: ascending order of country-level crop deficit; rank 3: maize, sorghum, millet, cassava, peanut; rank 4: peanut, cassava, millet, sorghum, maize.

\begin{tabular}{|l|l|l|l|l|}
\hline \multirow{2}{*}{ Country } & \multicolumn{4}{|c|}{ Future Coverage (\%) } \\
\cline { 2 - 5 } & Rank 1 & Rank 2 & Rank 3 & Rank 4 \\
\hline Benin & & & 59.65 & 58.35 \\
\hline Burkina Faso & 46.51 & 61.15 & 41.79 & 43.05 \\
\hline Gambia & 76.31 & 42.5 & 73.06 & 79.01 \\
\hline Ghana & 41.35 & 73.06 & 41.7 & 42.27 \\
\hline Guinea & 40.27 & 41.81 & 40.32 & 39.52 \\
\hline Guinea-Bissau & 38.45 & 40.29 & 38.03 & 37.96 \\
\hline Ivory Coast & 27.65 & 38.04 & 28.38 & 27.67 \\
\hline Mali & 12.00 & 27.52 & 11.02 & 11.96 \\
\hline Niger & 17.79 & 11.22 & 17.75 & 17.79 \\
\hline Nigeria & 84.46 & 83.65 & 83.27 & 85.91 \\
\hline Senegal & 45.78 & 51.69 & 49.82 & 46.57 \\
\hline Sierra Leone & 35.96 & 36.27 & 36.28 & 35.95 \\
\hline Togo & 51.53 & 50.93 & 52.73 & 53.18 \\
\hline
\end{tabular}




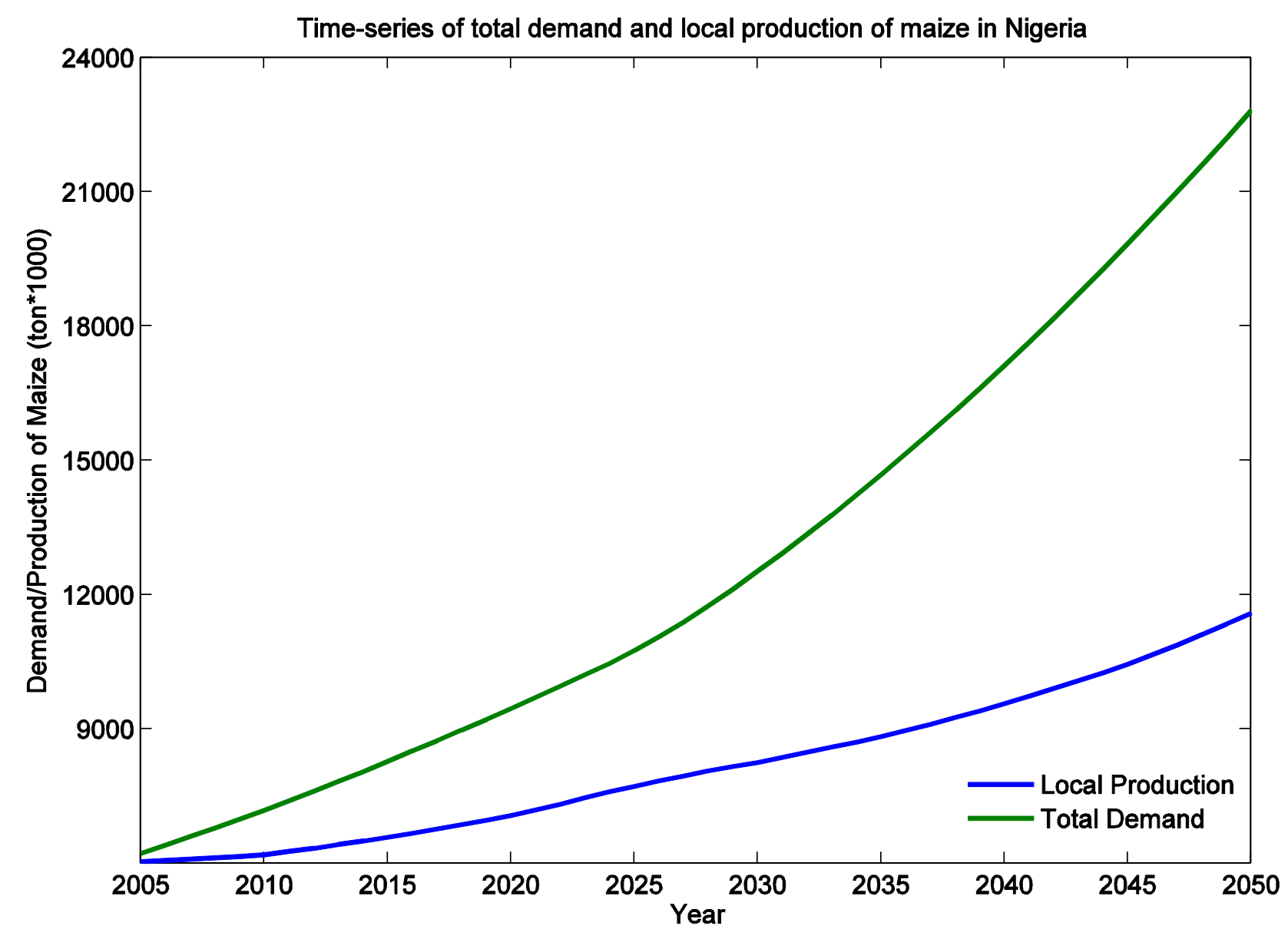

Figure S1: Time-series (2005-2050) of total demand and local production of maize in Nigeria according to future projection by the IMPACT model. 

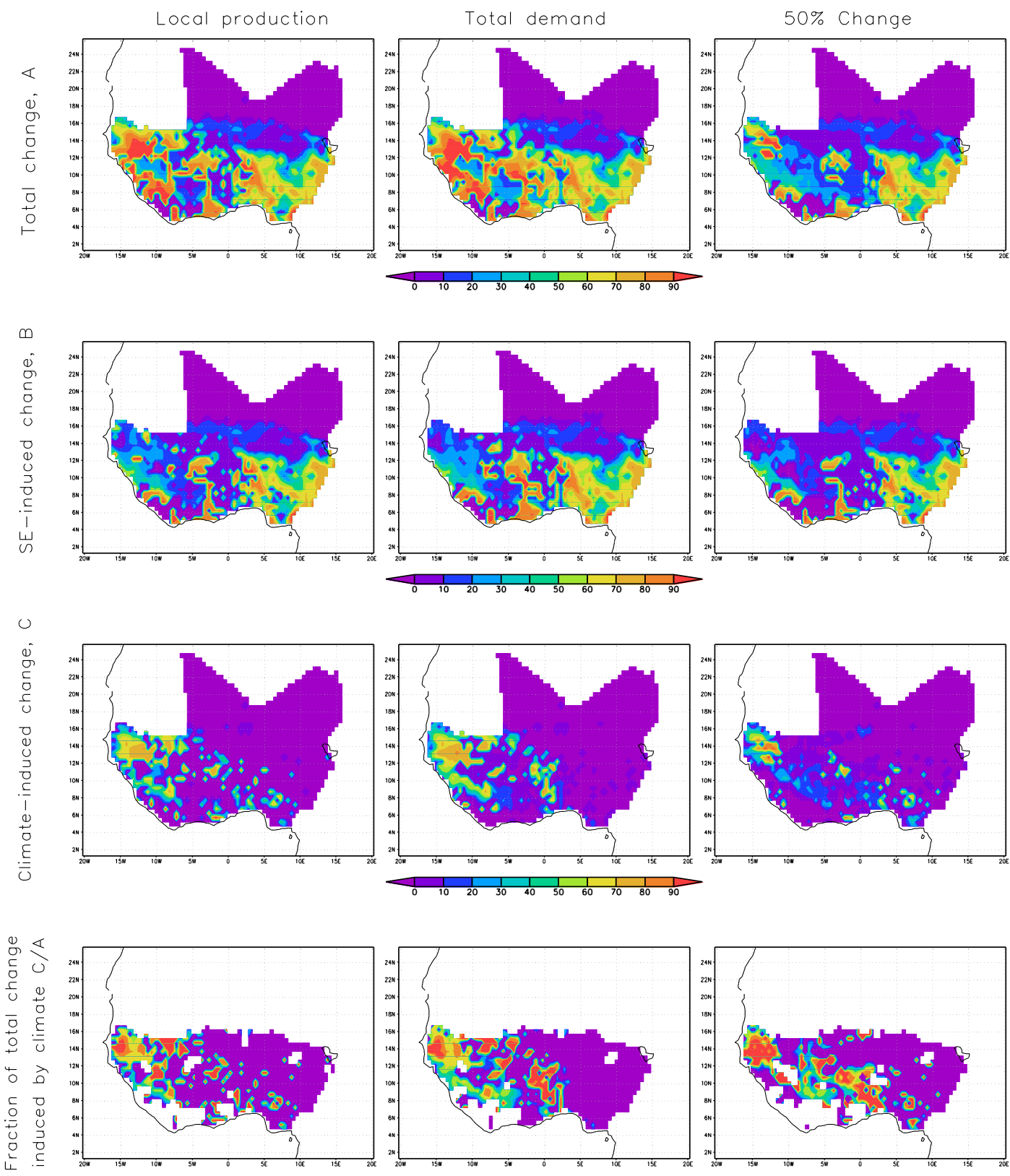

Figure S2: Sensitivity of land use change pattern to the demand values used as input to LandPro with the alternative cropping scenario following ascending order of yield under the MIROCdriven climate. $1^{\text {st }}$ row: absolute magnitude of total change for three future scenarios of demand; $2^{\text {nd }}$ row: change due to socioeconomic factors; $3^{\text {rd }}$ row: change due to climatic factors; $4^{\text {th }}$ row: fraction of climate-induced change to total change. 